\title{
Tendon-based design of wrist joint for tongue-controlled exoskeleton - a case study
}

\author{
Vestersøe, Kirstina B. Persson; Mohammadi, Mostafa; Kromann, Emil B.; Struijk, Lotte N. S. Andreasen
}

Published in:

Proceedings of 2021 leee 21st International Conference on Bioinformatics and Bioengineering

Link to article, DOI:

10.1109/BIBE52308.2021.9635558

Publication date:

2021

Document Version

Peer reviewed version

Link back to DTU Orbit

Citation $(A P A)$ :

Vestersøe, K. B. P., Mohammadi, M., Kromann, E. B., \& Struijk, L. N. S. A. (2021). Tendon-based design of wrist joint for tongue-controlled exoskeleton - a case study. In Proceedings of 2021 leee 21st International Conference on Bioinformatics and Bioengineering IEEE. https://doi.org/10.1109/BIBE52308.2021.9635558

\section{General rights}

Copyright and moral rights for the publications made accessible in the public portal are retained by the authors and/or other copyright owners and it is a condition of accessing publications that users recognise and abide by the legal requirements associated with these rights.

- Users may download and print one copy of any publication from the public portal for the purpose of private study or research.

- You may not further distribute the material or use it for any profit-making activity or commercial gain

- You may freely distribute the URL identifying the publication in the public portal 


\section{Tendon-based design of wrist joint for tongue- controlled exoskeleton - a case study}

\author{
Kirstina B. Persson Vestersøe \\ Department of Health Technology, \\ Technical University of Denmark, \\ Kgs. Lyngby, Denmark \\ kves0033@regionh.dk \\ Emil B. Kromann \\ Department of Health Technology, \\ Technical University of Denmark, \\ Kgs. Lyngby, Denmark \\ ebkro@dtu.dk/0000-0002-0782-1624
}

\author{
Mostafa Mohammadi \\ Department of Health Science and Technology, \\ Aalborg University, \\ Aalborg, Denmark \\ mostafa@hst.aau.dk / 0000-0002-5968-1248 \\ Lotte N. S. Andreasen Struijk \\ Department of Health Science and Technology, \\ Aalborg University, \\ Aalborg, Denmark \\ naja@hst.aau.dk /0000-0002-0484-5962
}

\begin{abstract}
Complete tetraplegia causes paralysis from the neck down, resulting in a need for constant care and lack of doing activities of daily living by yourself. Exoskeletons have enabled some rehabilitation and movement of paralyzed limbs but are often prohibitively large and heavy. This project aimed to redesign the wrist of the tongue controlled EXOTIC exoskeleton, making it compact and light. The new design used tendon-based technology, removing the motor from the forearm as well as employing 3D print. A proof-of-concept for the new design was obtained through a case study with three subjects.
\end{abstract}

Keywords - exoskeleton, tendon, tetraplegia, tongue control.

\section{INTRODUCTION}

Complete tetraplegia results in immobilization from the neck down. This decreases quality of life and incurs economic costs for constant help in activities of daily living (ADL) [1,2]. Spinal cord injury (SCI) is a global problem; with 250,000 to 500,000 affected individuals worldwide and an incidence between 40 and 80 per million, of which one-third are categorized as tetraplegia [2,3]. A study by Anderson uncovered that most individuals with tetraplegia would rather regain their arm movements than the movement of their legs, thus providing some autonomy in ADLs [4].

Some sensory and/or motor function can be retrained with support from exoskeletons [5]. Using exoskeletons to assist individuals with tetraplegia is complicated, however, by autonomic dysreflexia (AD), a comorbidity causing episodes of sudden hypertension with bradycardia, which can be fatal. While these episodes typically trace back to discomfort in the bladder, they can also be caused by tightly wrapped straps which is often deployed in exoskeletons [6].

Mechanisms for transmitting power from actuator to joint, divides exoskeletons into two groups: rigid and soft. Rigid exoskeletons have motors located on the joints and use gears to control torque. Soft exoskeletons use cables, or 'tendons', to transmit power to the joint. As tendon-based technologies tend to provide less control of force transmission, rigid designs are more common [5]. Rigid exoskeletons are, however, often large, and bulky, as exemplified by Gopura et al.'s SUEFUL-7 or Dezman et al.'s guided double rod exoskeleton $[7,8]$. The EXOTIC exoskeleton [9], discussed herein, represents yet another rigid exoskeleton that is relatively small in size compared to other rigid exoskeletons, e.g. [8], but incorporates larger parts such as the wrist rotation mechanism. EXOTIC is an upper-limb exoskeleton with five degrees of rotational freedom, including four motors located on the arm and a tendon-based glove (the BioServo ${ }^{\circledR}$ glove), actuated by a motor located next to the user $[9,10]$. The EXOTIC exoskeleton is unique in its incorporation of an intraoral tongue-computer interface (ITCI), which enables continuous and full control of all degrees of freedom through movement of the tongue $[11,12,13]$. Other control interfaces for assistive and rehabilitation exoskeletons are based on electromyography (EMG) [14,15], or electroencephalography (EEG) $[16,17,18]$, or other forms of brain-computer interfacing $[7,19]$.

Our aim in this study was to provide a small and lightweight exoskeleton, by reducing size and weight of the wrist-part of the EXOTIC exoskeleton. This was accomplished by moving actuators away from the arm and transmitting force via cables. We used 3D printing for producing light-weight alternatives to existing rigid components as in [20]. As detailed herein, a wristexoskeleton, controllable by the ITCI, was prototyped and performance-tested for ADLs, both as a stand-alone device and as part of the EXOTIC exoskeleton. A part of this paper is based on an unpublished report [21].

\section{Methodology}

Based on a literature study, we listed design requirements, as shown in Table I, and pursued a hybrid tendon-based solution, still using the ITCI control and the BioServo ${ }^{\circledR}$ glove, as shown in Fig. 1. This design is a lighter and less bulky alternative to the existing EXOTIC exoskeleton, where a relatively heavy motor and a $\mathrm{C}$-shaped rail is mounted on the elbow joint and forearm respectively, as shown in Fig. 2.

TABLE I: Requirements

Smaller and lighter than the existing EXOTIC solution

\section{Tendon-based}

Controllable by ROS and the ITCI control

Compatible with the upper arm and elbow joint of the existing EXOTIC exoskeleton

Must not harm the user

Can hold and move items up to $0.5 \mathrm{~kg}$

Includes encoders for position/rotation feedback 


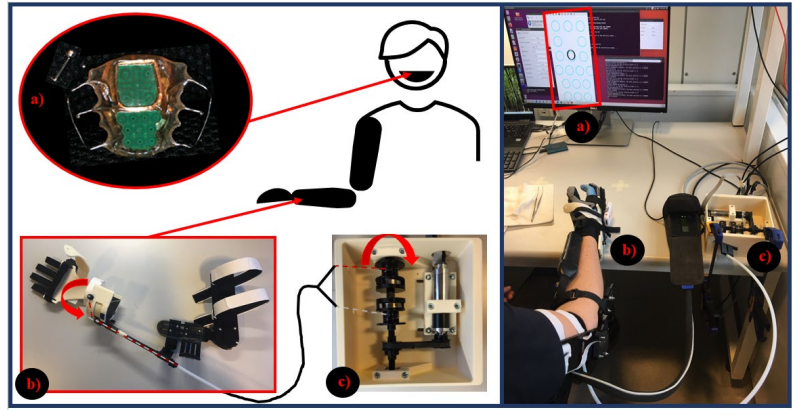

Fig. 1: The new design. a) The ITCI control: A retainer and a ferromagnetic tongue piercing, and the associated graphical user interface. b) The full design of the proposed wrist exoskeleton, which merged the hand and wrist parts and connected the device at the elbow. The upper arm brace, fastened by straps, provided a stand-alone option. c) The motor box containing a 4-pole MAXON motor. The belt drive transferred torque to the shaft, where two custom-made pulleys controlled the wrist-rotation, by rotating in opposite directions while keeping both cables taut (the red arrows).

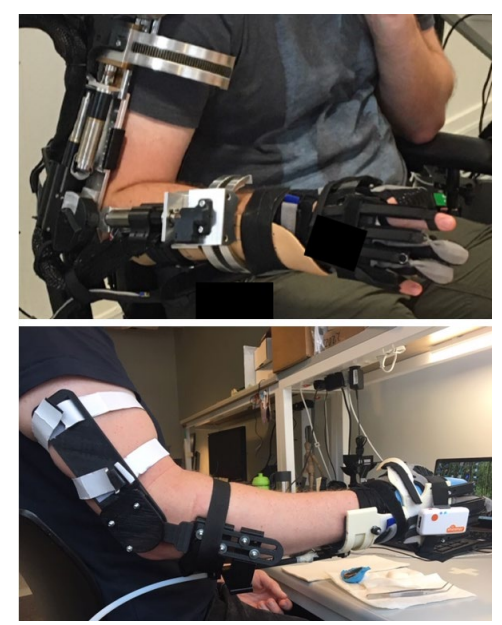

Fig. 2: Comparison: top) The existing EXOTIC exoskeleton, bottom) The new design proposed in this study.

\section{A. Combined hand and wrist}

We implemented a combination of the existing EXOTIC exoskeleton hand part, supporting the BioServo ${ }^{\circledR}$ glove, and a new wrist part. As the existing hand part was $3 \mathrm{D}$ printed, the wrist part was readily designed to mate to the hand part in CAD software (Fusion 360), thus, avoiding extra strappings to secure the device. Our new design used a U-shaped wrist brace, which only required strappings of the BioServo ${ }^{\circledR}$ glove to fixate the wrist of the user. The new wrist part was relatively compact, as it only needed to facilitate rotation, and did not carry the weight of the forearm. An external rail on the Ushaped wrist part enabled wrist-rotation about the axis of the forearm. Specifically, a ball bearing locked by two holders, moved the wrist part from supination to pronation, by two 'tendons' attached to both sides, as shown in Fig. 3.

Force for the rotation was transmitted via two tendons, here fishing lines, which could pull in opposite directions. To reduce wiring-complexity the tendons were collected in a hollow rod. Physical stops, $180^{\circ}$ apart on the U-shaped rail, ensured that the wrist could not overstretch and harm the user. The size of the ball bearing, however, decreased the range of motion (ROM) to $146^{\circ}$, or $\pm 73^{\circ}$, from the neutral $0^{\circ}$ angle, (the 'thumbs up' position). For additional safety, stops to prevent overstretching were also set in the control software.

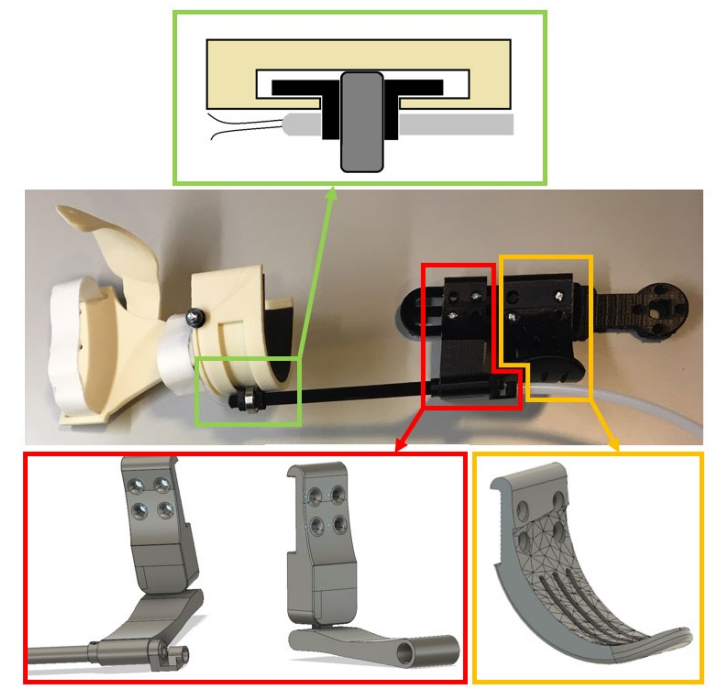

Fig. 3: The design of the wrist exoskeleton. Top) the green box schematizes the rail on the wrist part (yellow). The ball bearing (dark gray) is kept in place by two holders (black) and a rod (light gray). The wrist rotates when a tendon is pulled. The rod connects the elbow and wrist, while holding the ball bearing system in place and routing the tendons. Bottom) The elbow connection of the new design. The red box highlights the passive joint and the locking mechanisms of the rod. The orange box highlights the elbow rest, which was a part of the elbow joint design of the existing EXOTIC exoskeleton.

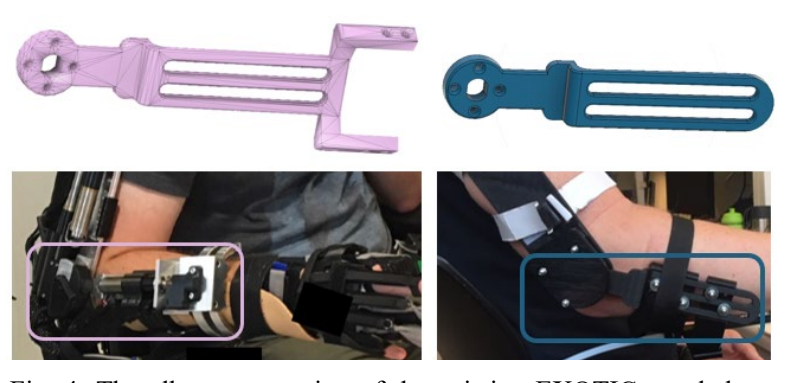

Fig. 4: The elbow connection of the existing EXOTIC exoskeleton and the new design. The existing design was shaped as a fork (pink) to carry the motor and $\mathrm{C}$-shaped wrist rail. The new design (blue) was shorter, as it only carried the elbow rest and the passive joint.

\section{B. Elbow attatchment}

The elbow of the existing EXOTIC exoskeleton was a controllable, one-degree-of-freedom (DoF) joint. As shown in Fig. 4, the ring-shaped connection at the elbow joint of the existing EXOTIC exoskeleton was reproduced in our new design, thus maintaining compatibility. However, a passive elbow joint was used during concept-testing.

To test the new design as a stand-alone tool, we produced an upper arm brace, as shown in Fig. 5. This design would be useful for users with incomplete damage at C5 level or lower, i.e., individuals who can move their shoulders. Two separate parts were attached, as shown in Fig. 3. First, the elbow brace, located closest to the joint, which carried the weight of the forearm and hand. Secondly a passive joint attaching the rod from the wrist part to the elbow.

A Bowden tube routed tendons from the motor box into the rod, see Fig. 1. A grove underneath the rod ensured that the ball bearing part and associated holders (Fig. 3) would not rotate about the rod itself. The main function of the holders was to keep the ball bearing in place. The design of the holders is shown in Fig. 6. 


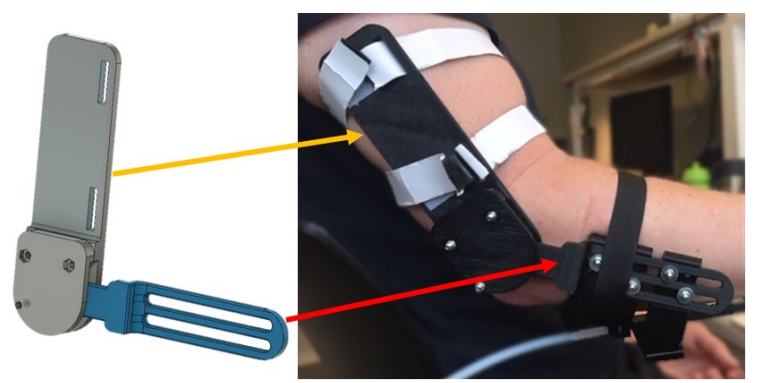

Fig. 5: Brace connecting the elbow joint and exoskeleton to the user. New elbow connection (red arrow). Upper arm brace (orange arrow).

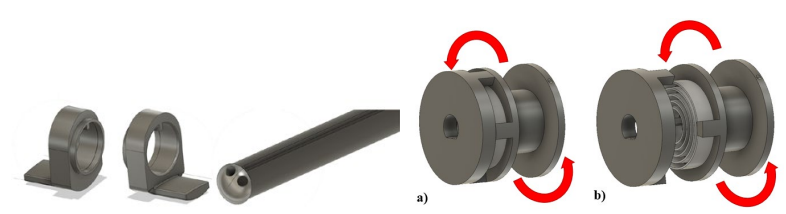

Fig. 6: Top) Holders keeping the ball bearing in place. Holes for the tendons and a grove (for the keys on the holders) is visible on the rod. Bottom) The pulleys in the motor box. The design uses a spiral spring to keep tendons taut. a) The large part of the pulley is rotated due to tension in the tendons. When this tension is released, a spring pulls the tendons back. b) The same pulley slightly disassembled.

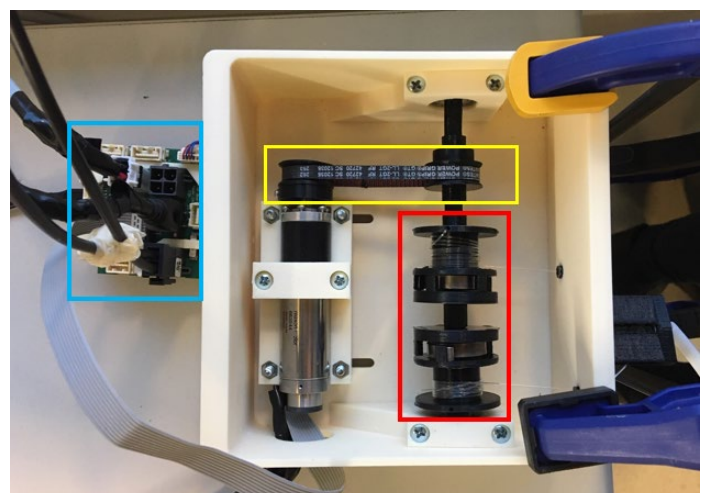

Fig. 7: The motor box. Yellow) A belt transfers torque from the motor to the shaft. Red) Two pulleys on the shaft, control wrist rotation. Blue) Motor controller and associated cables.

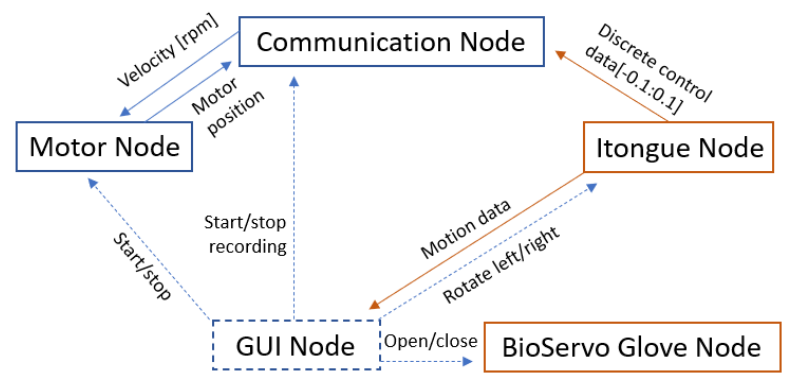

Fig. 8: Communication of the ROS nodes. Blue nodes were written as part of this work, while orange nodes already existed in the EXOTIC exoskeleton setup. The node with a dashed outline was modified from existing code to integrate the new design and facilitate case studies.

\section{Motor box}

The motor box housed a MAXON EC 4 pole motor with a planetary reduction gear (factor 3.8) attached to a belt. The belt transferred torque from the motor to a shaft with ball bearings at each end. 3D printed pulleys were designed in two parts and used spiral springs, ensuring constant tension on the attached tendons, see Fig. 6 . One part was attached to the shaft, creating limits for the spring. The other was attached to the spring, and otherwise rotating freely. The motor box and the wrist part were printed in ASA (Stratasys Fortus 380mc). All other parts were printed in PLA (Ultimaker 2). The motor box, measuring $156 \times 158 \times 70 \mathrm{~mm}$, is shown in Fig. 7 .

\section{Software control}

The motor was controlled with a EPOS4 digital positioning controller and a PC running Robot Operating System (ROS) on Ubuntu. A node written in C++ controlled the motor, while nodes written in Python enabled control of the wrist exoskeleton and the BioServo ${ }^{\circledR}$ glove through the ITCI, as well as adding a graphical user interface (GUI), see Fig. 8.

\section{E. ITCI control and GUI}

The ITCI system consists of a dental retainer with 18 inductive coils, an activation unit (AU) mounted on the tongue, and a central unit outside the oral cavity [12], see Fig. 1. The ITCI used in this study was an adapted version the commercially available system (Itongue ${ }^{\circledR}$, TKS, Denmark) [11]. The coils are used to track the position of the AU. In this work we mapped positions across the retainer to exoskeleton motions, as shown in Fig. 9. For our case studies, we used an ITCI mouthpiece embedded in a dental putty, and AUs which were glued to participants' tongues with tissue glue.

\section{F. Case study}

We aimed to test the ADL-functionality of the new wrist exoskeleton and to confirm that our design requirements were met. Our case study included three healthy male subjects, age 28-30, who had prior experience with ITCI. The study evaluated four tasks:

(1) ROM task, where the full ROM of $146^{\circ}$ was tested.

(2) Bottle task, where a bottle was grasped, moved, and rotated to pour water into a cup. The cup was located $27 \mathrm{~cm}$ from the bottle, and the bottle was positioned $30 \mathrm{~cm}$ away from the subject. The bottle was then put down. Due to complications with sliding in the driving belt, the two first subjects completed this 'task 2' with a small amount of water. The issue was fixed for the third subject, who completed the task with $500 \mathrm{ml}(0.5 \mathrm{~kg})$.

(3) Strawberry task, where a plastic strawberry was lifted from the table and moved to the subject's mouth. Next, the item was returned using rotation in different segments.

(4) Accuracy task, where movement started by rotating to $-45^{\circ}$ and then to $+45^{\circ}$ before finally rotating to $0^{\circ}$. The subject observed the angle as markings on the wrist. Two of the three subjects used a mirror for a better view.

All tasks were repeated five times. The study was approved by the National Committee on Health Research Ethics (Denmark).

\section{G. Data acquisition and analysis}

Motor position and ITCI data were collected in ROS version 16.04 LTS, using ROS bag, and extracted as csv files. Data analysis was conducted in MATLAB version R2020a. Some design optimization was performed between trials with different subjects. Hence, results are not readily comparable between subjects. For all subjects, the starting time for a given task was defined as the beginning of motion, thus making the recorded data more comparable. 


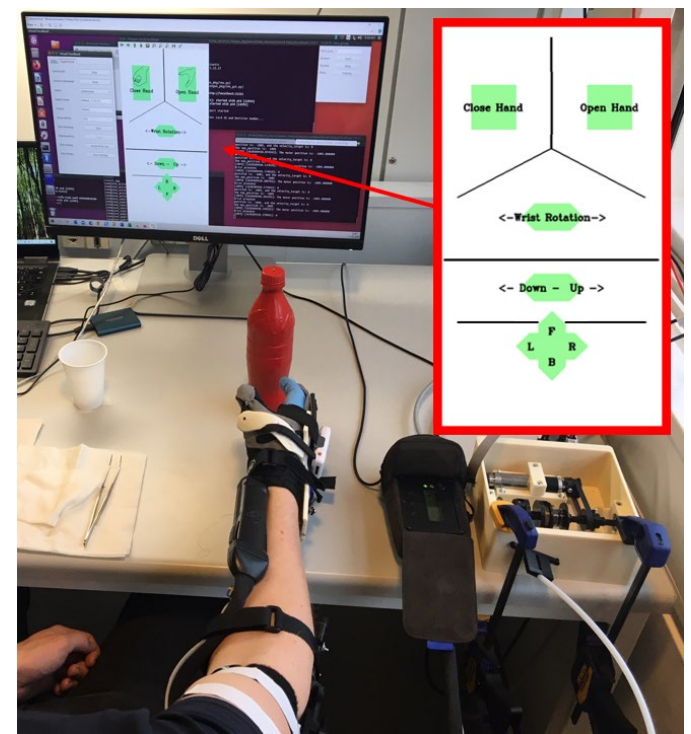

Fig. 9: The case study (specifically, the bottle task). The setup included the BioServo ${ }^{\circledR}$ glove and the tongue control layout in the red box.

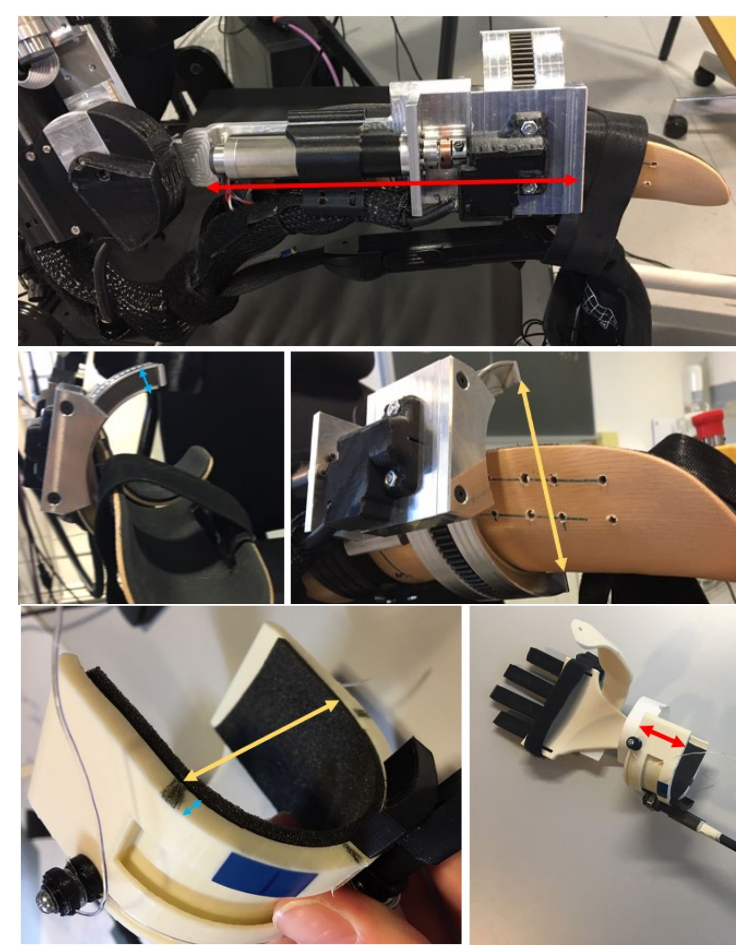

Fig. 10: The measured entities referenced in Table II, red is the length, blue is the width, and yellow is the diameter of the C-shape and Ushape, respectively. The top three images show the EXOTIC design, and the bottom two images show the new design.

TABLE II: Exoskeleton measurements

\begin{tabular}{c|c|c|c}
\hline \hline & The EXOTIC design & The new design & Diff. \\
\hline Length & $185 \mathrm{~mm}$ & $40 \mathrm{~mm}$ & $21.6 \%$ \\
\hline Width & $30 \mathrm{~mm}$ & $5 \mathrm{~mm}$ & $16.7 \%$ \\
\hline Diameter & $130 \mathrm{~mm}$ & $65 \mathrm{~mm}$ & $50.0 \%$ \\
\hline ROM & $130^{\circ}$ & $146^{\circ}$ & $12.3 \%$ \\
\hline \hline
\end{tabular}

\section{RESULTS}

\section{A. The exoskeleton design}

Using a U-shape instead of a C-shape enabled the wrist to move closer to the center of rotation of the forearm and, thus, allowed a more compact design, see Fig. 10 and Table II. No weight measurements were made. Yet, given the significant size-reduction and a future use of the same material as the existing exoskeleton, we anticipate a corresponding weight reduction. The new U-shape of the wrist part enabled a ROM of $146^{\circ}$, see Table II, while the existing wrist part of the EXOTIC exoskeleton only allows a ROM of $130^{\circ}$. The builtin rail also ensured physical barriers for the wrist rotation, protecting the user. The new connection in the elbow joint performed well; the passive joint of the upper arm brace enabled movement for ADLs, and the connection carried the elbow rest and the passive joint. The passive joint was, however, not completely robust. Small deviations from a $90^{\circ}$ angle between the ball bearing and the passive joint caused friction, leading to control problems. Connecting the hand and wrist parts reduced the need for strappings, as the hand and wrist parts shared the same strappings around the wrist.

\section{B. Case study: The four tasks}

The three subjects repeated the four tasks five times. Data from all these (60) trials are shown in Fig. 11. The first task examined the full ROM, the second, as well as the third task examined the functionality of the exoskeleton regarding ADLs and the fourth task examined the precision of the ITCI control. Some of this data is, however, difficult to assess due to sliding and displacement of the motor.

\section{Case study: The requirements}

The wrist exoskeleton was controlled using interfaces equivalent to (derived from) the existing EXOTIC system. Some new ROS nodes enabled satisfactory control of the wrist exoskeleton through the ITCI system. The new code contained a homing function as well as software-boundaries, which referenced the motor position, rather than angleencoders in the exoskeleton (likely, a better solution). Using the EPOS Studio software for the MAXON motor, the movement limits were set to \pm 2380 increments, i.e., just before hitting the physical stops in the U-shaped rail. While these safety measures did work, sliding of the belt in the motor box caused a gradually increasing mismatch between the wrist angle 'assumed' by software and the actual angle. This error manifests in multiple trials as flat lines (where further motion is halted by software), even inside the safe ranges of wrist rotation.

An important requirement for the functionality was the ability to hold a $0.5 \mathrm{~kg}$ payload. This was tested and failed for the first subject due to slipping of the belt drive, prohibiting further rotation of the wrist. The pulleys were redesigned and fastened with setscrews, which resulted in a significant improvement. The third subject completed the task successfully, with rotation of the $0.5 \mathrm{~kg}$ payload.

During experiments with all three subjects, we faced challenges with the coded rotation-limits, see Fig. 11, due to sliding of the driver belt and pulleys (first and second subject). For the first subject the software boundaries controlling the motor were increased to be negligible, due to the excessive motor sliding issue, which was evident from the large deviation in the motor position data. 

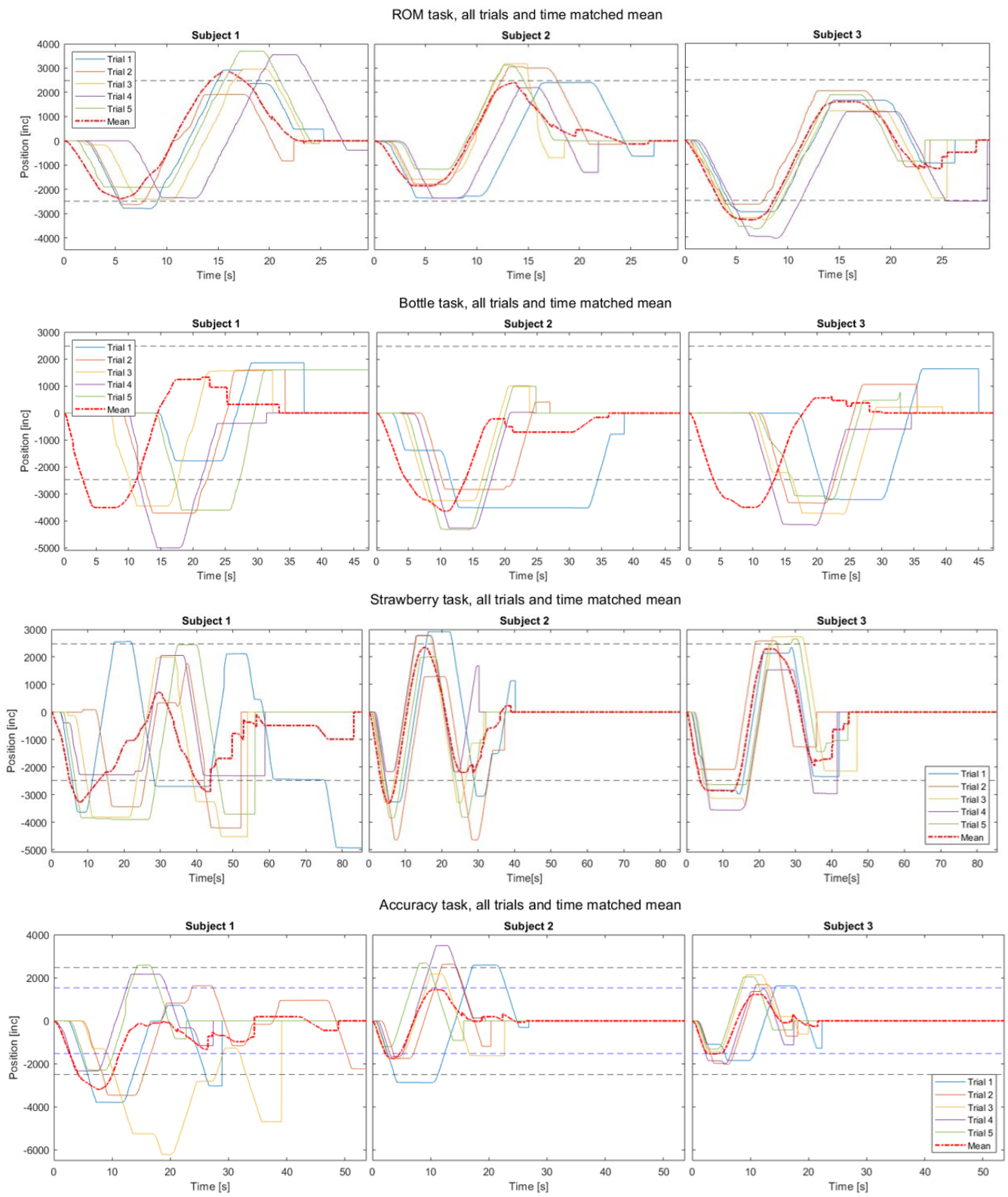

Fig. 11: Row 1) The ROM task for all subjects. Row 2) Bottle task, Row 3) Strawberry task, Row 4) Accuracy task. All five trials are displayed alongside their time-matched mean (dashed red line). Dashed blue lines indicate $\pm 45^{\circ}$ rotation, and dashed black lines indicate coded boundaries

\section{DISCUSSION}

\section{A. The built design}

Table II showed a significant decrease of the size of the new design by $16.7-50.0 \%$ compared the existing EXOTIC solution. The main differences were the repositioning of the motor from the forearm to the motor box, and the change from $\mathrm{C}$-shape to a U-shaped guiding rail. The existing EXOTIC solution is built from aluminum and brass, this will also be the case for the next iteration of the new design. Yet, a few parts, such as the wrist, will be advantageous to keep as 3D print or carbon reinforced plastics for easy sizing and low weight. The U-shaped wrist resulted in a ROM of $146^{\circ}$, which is an increase of $12.3 \%$. This is critical as ROM is an important parameter for ADLs.

The tendons were non-elastic fishing line, with a diameter of $0.7 \mathrm{~mm}$, which easily bent into the necessary angles of the pulley system and the wrist part. The tendons facilitated the move of the motor to the adjacent motor box, which can be placed e.g., on the user's wheelchair.

Due to risk of $\mathrm{AD}$, strappings were kept at a minimum by combining the hand and wrist parts. Our case study, nonetheless, implemented strappings in regard to the glove, the hand/wrist part, and the upper arm brace to keep the device in place, see Fig. 9. To find a solution with less strapping, the users should be involved in the design, to ensure the best comfort and a better understanding of the individual AD causes.

For the design of the rail, using a ball bearing kept in place by two holders, friction was an obvious challenge. The solution was to use ASA plastic for the wrist part and adding lubrication. The holders were designed for the curve of the rail, while the ball bearing carried the force, see Fig. 3 .

The main challenge encountered in our case study was slipping of the belt drive. Better fastening of the pulleys in the 
motor box helped. Likely, replacing the belt with gears would be even better. Deviations in the angles of the ball bearing and the shaft in the motor box caused some friction. Hence, we recommend implementing a more robust solution in future iterations of the design.

\section{B. The case study}

For all subjects, the sliding of the driving belt resulted in incorrect motor position data, which was clear in Fig. 11. The error-prone position data had no consequence for the users, due to the physical barriers of $\pm 73^{\circ}$.

The ROM task, see Fig. 11 (top row), showed similar motion patterns for the subjects when examining the mean. The motion patterns also illustrate the versatility of tongue control, as we only observe smooth motion patterns.

The bottle task and the strawberry task, see Fig. 11 (middle rows), examined the performance of ADLs. These tasks involved the BioServo ${ }^{\circledR}$ glove, which was activated by the ITCI. Challenges with the sliding belt drive were seen, but a positive progression was evident as the problem was solved. The bottle task was for the first and second subject conducted with a small amount of water. The third subject could, however, successfully complete the task with $500 \mathrm{ml}(0.5 \mathrm{~kg})$ water. When flexing the elbow during the strawberry task, the angle between the wrist part and the rod caused friction in the wrist rail. Subjects intuitively solved this issue, by rotating the strawberry before the arm was raised towards the face.

The accuracy task examined the precision of the tongue control, see Fig. 11 (bottom row). While subject 1 needed several position adjustments to complete the task, subjects 2 and 3 needed no (or few) adjustments during the test. This difference in performance aligns with the belt drive being more stable for subjects 2 and 3 .

The software boundaries and the homing function were derived from the starting position of the wrist exoskeleton and the motor position. If these were incorrect, the control was dysfunctional. Likely, angle-encoders directly on the wrist joint can solve this problem.

\section{CONCLUSION}

This study produced a compact and light exoskeleton design for wrist rotation, controllable by the modified ITCI of the EXOTIC exoskeleton (16.7-50.0\% size reduction compared to the existing EXOTIC exoskeleton). Multiple requirements were set to ensure the performance, and most were met. Due to physical barriers, the wrist exoskeleton could not harm the user, however, due to motor sliding, the software boundaries proved unreliable. In future work, we recommend replacing the belt drive by more robust gears and we recommend using stronger and more stable materials. Our case study demonstrated successful exoskeleton-control via the tongue interface. The design proposed in this study is compatible with the existing EXOTIC exoskeleton and can be used as a stand-alone device.

\section{ACKNOWLEDGMENT}

A kind thank you to the EXOTIC research group members; Mikkel Thøgersen and Stefan Hein Bengtson.

\section{REFERENCES}

[1] National Spinal Cord Injury Statistical Center. "2020 Annual Statistical Report for the Spinal Cord Injury Model Systems”, University of Alabama at Birmingham. [Online]. Available at: https://www.nscisc.uab.edu (accessed 09.10.2021)

[2] National Spinal Cord Injury Statistical Center, "Facts and Figures at a Glance”, University of Alabama at Birmingham, (2021).

[3] World Health Organization, "Spinal Cord Injury” [Online]. Available at: https://www.who.int/news-room/fact- sheets/detail/spinal-cord-injury (accessed 23.09.2020)

[4] K. D. Anderson, "Targeting recovery: Priorities of the spinal cordinjured population”, Journal of Neurotrauma, vol. 21, pp. 1371-1383 (2004)

[5] M. A. Gull, et al., “A review on design of upper limb exoskeletons Robotics”, vol. 9 (2020), DOI: 10.3390/robotics9010016

[6] K. C. Eldahan, A. G. Rabchevsky, "Autonomic dysreflexia after spinal cord injury: Systemic pathophysiology and methods of management", Autonomic Neuroscience, vol. 209, pp. 59-70 (2019),

DOI: 10.1016/j.autneu.2017.05.002

[7] R. A. R. C. Gopura, K. Kiguchi and Y. Li, "SUEFUL-7: A 7DOF upper-limb exoskeleton robot with muscle-model-oriented EMG-based control," 2009 IEEE/RSJ International Conference on Intelligent Robots and Systems, 2009, pp. 1126-1131, doi: 10.1109/IROS.2009.5353935.

[8] M. Dezman, et al., "Exoskeleton arm pronation/supination assistance mechanism with a guided double rod system”, IEEE-RAS 19th International Conference on Humanoid Robots (2019)

[9] M. Thøgersen, et al., "EXOTIC - A Discreet User-Based 5 DoF UpperLimb Exoskeleton for Individuals with Tetraplegia," 2020 3rd International Conference on Mechatronics, Robotics and Automation (ICMRA), pp. 79 83, (2020), DOI: 10.1109/ICMRA51221.2020.9398351

[10] Bioservo, Carbonhand [Online]. Available at: https://www.bioservo.com/healthcare/carbonhand (accessed 23.09.2020)

[11] TKS Technology, Om TKS [Online]. Available at: https://tkstechnology.dk/om-tks/ (accessed 12.10.2020)

[12] L. N. S. A. Struijk, et al., "Development and functional demonstration of a wireless intraoral inductive tongue computer interface for severely disabled persons", Disability and Rehabilitation: Assistive Tech., vol. 12, no. 6. (2016)

[13] M. Mohammadi, et al., "Eyes-free gesture based tongue control of a five DOF upper-limb exoskeleton for severely paralyzed individuals", Frontiers in Neuroscience, in press (2021)

[14] C. Fleischer, et al., Application of EMG signals for controlling exoskeleton robots, Biomed Tech (2016)

[15] DR. L. F. Abusedra, H. F. Busedra, M. Elzawi, "Lower limb exoskeleton control using EMG signal analysis", In Proceedings of the 6th International Conference on Engineering \& MIS 2020 (ICEMIS'20). Association for Computing Machinery, Article 95, pp. 1-7. (2020), DOI: $10.1145 / 3410352.3410831$

[16] A. C. Villa-Parra, et al., "Towards a robotic knee exoskeleton control based on human motion intention through EEG and sEMG signals", 6th Intl. Conference on Applied Human Factors and Ergonomics (2015) [17] C. Wang, et al., "Implementation of a brain-computer interface on a lower-limb exoskeleton”, IEEE Access Special Section on Cloud-based Robotic Systems for Intelligent Services (2018)

DOI: 10.1109/AC- CESS.2018.2853628

[18] N.-S. Kwak, et al., “A lower limb exoskeleton control system based on steady state visual evoked potentials", Journal of Neural Engineering, vol. 12 (2015), DOI: 10.1088/1741-2560/12/5/056009

[19] A. L. Benabid, et al., "An exoskeleton controlled by an epidural wireless brain-machine interface in a tetraplegic patient: a proof-ofconcept demonstration”, Lanchet Neural, vol. 18, pp. 1112-1122 (2019) DOI: 10.1016/S1474- 4422(19)30321-7

[20] H. Dodziuk, "Applications of $3 D$ printing in healthcare", Kardiochirurgia i torakochirurgia polska $=$ Polish journal of cardio-thoracic surgery vol. 13.3, pp. 283-293, (2016), DOI:10.5114/kitp.2016.62625 [21] K. B. P. Vestersøe, et al., Tendon-based design of wrist joint for tongue-controlled exoskeleton, Unpublished thesis (2021)

[22] M. Mohammadi, "Eyes-free gesture based tongue control of a five dof upper-limb exoskeleton for severely paralyzed individuals," submitted to Frontiers in Neuroscience, 2021. 